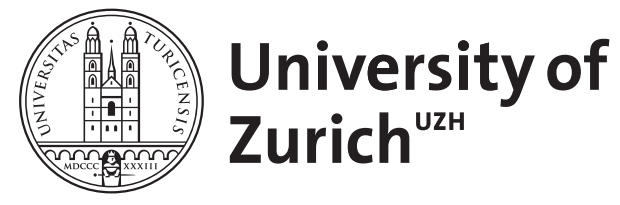
Archive

University of Zurich

University Library

Strickhofstrasse 39

CH-8057 Zurich

www.zora.uzh.ch

Year: 2013

Thromboseprophylaxe nach Hüftgelenkschirurgie - Aspirin ist Heparin nicht unterlegen

Markun, Stefan

DOI: https://doi.org/10.1024/1661-8157/a001412

Other titles: Thrombosis prophylaxis after hip surgery- aspirin is not inferior to heparin

Posted at the Zurich Open Repository and Archive, University of Zurich

ZORA URL: https://doi.org/10.5167/uzh-90676

Journal Article

Accepted Version

Originally published at:

Markun, Stefan (2013). Thromboseprophylaxe nach Hüftgelenkschirurgie - Aspirin ist Heparin nicht unterlegen. Praxis, 102(21):1323-1324.

DOI: https://doi.org/10.1024/1661-8157/a001412 


\title{
Thromboseprophylaxe nach Hüftgelenkschirurgie - Aspirin ist Heparin nicht unterlegen
}

\author{
Frage: \\ Was ist die Wirksamkeit und Sicherheit von Aspirin verglichen mit niedermolekularem Heparin \\ (NMH) in der Thromboseprophylaxe nach totaler Hüftarthroplastie?

\section{Hintergrund:} \\ Nach orthopädischen Eingriffen am Hüftgelenk besteht ein hohes Risiko für venöse \\ Thrombosen. Eine entsprechende Prophylaxe ist meist indiziert. Bislang wurden Heparine oder \\ Vitamin-K Antagonisten verwendet. Vermehrt werden mittlerweile die neuen oralen \\ Antikoagulantien eingesetzt, welche sich durch geringen Aufwand sowohl in der \\ Verabreichung, als auch im Kontrollbedarf auszeichnen. Aspirin hat ebenfalls diese \\ Eigenschaften, zudem zu einem Bruchteil der Kosten. Die Verwendung von Aspirin als \\ Thromboseprophylaxe nach Hüftgelenksersatz wird jedoch sehr kontrovers beurteilt. Diese \\ Studie vergleicht Aspirin mit niedermolekularem Heparin (NMH) bezüglich des Auftretens von \\ venösen Thrombosen und Blutungskomplikationen nach totaler Hüftarthroplastie.
}

\section{Einschlusskriterien:}

- Patienten die sich einer elektiven, einseitigen totalen Hüftarthroplastie unterzogen (primäre Arthroplastien und sekundäre Formen)

\section{Ausschlusskriterien:}

- Hüftfraktur innerhalb der vergangen 3 Monaten

- Metastasierendes Malignom, Lebenserwartung < 6 Monate, Blutung mit Kontraindikation für Antikoagulantien, aktives peptisches Ulkus oder Gastritis mit Kontraindikation für Aspirin, Allergie gegen Aspirin, Heparin induzierte Thrombopenie oder Allergie gegen Heparin, Kreatininclearance $<30 \mathrm{ml} / \mathrm{min}$, Thrombozyten $<100 \mathrm{G} / \mathrm{L}$, Indikationen für Langzeitantikoagulation, postoperative tiefe Venenthrombose vor Randomisierung

\section{Studiendesign und Methode:}

Randomisierte, doppelblinde Multizentrumsstudie. Die Operationstechniken wurden individuell von den Operateuren festgelegt. Während der ersten 10 Tage postoperativ erhielten alle Patienten 5000I.E. NMH s.c. einmal täglich. Zwischen Tag 8 und Tag 10 wurde randomisiert, ab Tag 10 wurden die Interventionen gestartet.

\section{Studienort:}

12 Universitätsspitäler in Kanada

\section{Interventionen:}

- NMH Gruppe: 28 Tage einmal täglich 5000 I.E. NMH s.c. plus Placebo p.o.

- Aspirin Gruppe: 28 Tage einmal täglich Placebo s.c. plus $81 \mathrm{mg}$ Aspirin p.o.

\section{Outcome:}

\section{Primärer outcome}

- Wirksamkeit: Auftreten von tiefen Venenthrombosen und Lungenembolien innerhalb von 90 Tagen nach Randomisierung (bestätigt durch einen objektiven Test)

- Sicherheit: Grössere Blutungen (Blutungen, die tödlich verliefen, symptomatisch waren, in kritischer Region oder Organen stattfanden, einen Hämoglobinabfall von $2 \mathrm{~g} / \mathrm{dl}$ oder mehr bewirkten oder die Gabe von 2 oder mehr Vollbluttransfusionen erforderten) 


\section{Sekundäre outcomes}

- Todesfälle, klinisch relevante kleinere Blutungen (Blutungen, die zu Hospitalisierung, oder infizierten Hämatomen führten, oder welche eine Reoperation oder Aspiration erforderten), sonstige kleinere Blutungen, Myokardinfarkte, cerebrovaskuläre Insulte, Wundinfekte

\section{Resultat:}

- 786 Patienten konnten randomisiert werden (400 zu NMH und 386 zu Aspirin). Die Patienten waren durchschnittlich 58 Jahre alt und zu 57\% Männer, 80\% der Patienten erhielten eine primäre totale Hüftgelenksarthroplastie.

- Unter NMH hatten 5 Patienten (1.3\%) ein venös-thrombotisches Ereignis innerhalb von 90 Tagen, unter Aspirin 1 Patient (0.3\%). Statistisch bedeutet dies, dass Aspirin nicht unterlegen war, jedoch auch nicht signifikant überlegen.

- Unter NMH trat eine einzige grössere Blutung auf, unter Aspirin keine. Klinisch relevante kleinere Blutungen traten unter $\mathrm{NMH}$ in 4 Fällen auf, unter Aspirin in 2. Sonstige kleinere Blutungen traten unter NMH in 18 Fällen auf (4.5\%), unter Aspirin in 8 Fällen (2.1\%).

- 39 Patienten in der Studie hatten Aspirin gleichzeitig als Langzeitmedikation verordnet. Diese Patienten standen in der Studie somit entweder unter Aspirin plus NMH, oder unter doppeltem Aspirin. Die separate Analyse dieser Fälle ergab keine thrombotischen Ereignisse, ein Patient, welcher doppelt Aspirin erhalten hatte erlitt eine kleinere klinisch relevante Blutung.

\section{Kommentar:}

- In dieser Studie unterschied sich Aspirin nicht signifikant von Heparin bezüglich des Verhinderns von venösen Thrombosen oder Blutungskomplikationen ab dem zehnten Tag nach Hüftgelenksarthroplastie. Die Autoren weisen aber auf einen Trend hin, der Aspirin in beiden Belangen favorisiert.

- Die Studie musste mit einer geringeren Fallzahl auskommen als ursprünglich geplant war: Während der Rekrutierungsphase wurde Rivaroxaban zugelassen, was die Rekrutierung stark erschwerte, da viele Orthopäden davon abkamen NMH zu verschreiben. Die vorgezogene Analyse der Resultate zeigte jedoch, dass die Nicht-Unterlegenheit bereits anhand der bislang untersuchten Fälle bestätigt werden konnte, worauf entschieden wurde die Rekrutierung vorzeitig zu beenden.

- Die Compliance bei der Selbstmedikation könnte bei den subkutanen Präparaten schlechter gewesen sein, als bei den Tabletten (ungeachtet ob Verum oder Placebo). Dies könnte zu einem verzerrenden Effekt zugunsten von Aspirin geführt haben, da somit Aspirin tendenziell mit Placebo verglichen worden wäre.

- Das Aufkommen der neuen oralen Antikoagulantien hat diese Studie sozusagen eingeholt. Ein Vergleich mit diesen Produkten wäre ebenfalls sehr interessant gewesen. Direkte Vergleiche von Wirksamkeit und Sicherheit der neuen oralen Antikoagulanzien verglichen mit Aspirin sind aus dieser Studie nicht möglich, die Frage nach den direkten Behandlungskosten allerdings wäre bereits eindeutig zu beantworten.

\section{Literatur:}

Anderson DR, Dunbar MJ, Bohm ER, et al. Aspirin versus low-molecular-weight heparin for extended venous thromboembolism prophylaxis after total hip arthroplasty: a randomized trial. Ann Intern Med. Jun 2013;158(11):800-806.

\section{Verfasser:}

